\title{
Estudo Dos Exames Complementares Solicitados Pela Atenção Básica Em Saúde Em Um Município De Grande Porte
}

\author{
Study Of The Complementary Tests Requested By The Primary Health Care In A Large \\ Municipality
}

Estudio De Los Exámenes Complementarios Solicitados Por La Atención Básica En Salud En Municipio De Gran Porte

Ricardo Machado Castanheira de Souza ${ }^{1}$ Amanda Frias ${ }^{2}$ Nivaldo Carneiro Junior $^{3}$

\section{Resumo}

A solicitação não criteriosa de exames complementares nas consultas médicas tem se tornado incompatível com a disponibilidade do Sistema Único de Saúde (SUS), gerando agendamento a longo prazo, exigindo estratégias de intervenção. Nessa direção, surge o Programa "Corujão da Saúde", em 2017, da Secretaria Municipal da Saúde de São Paulo (SMS/SP), visando zerar as filas de espera de determinados exames solicitados na rede de atenção básica. Este trabalho, transversal e quantitativo, caracterizou a pertinência dessas solicitações. Analisaram-se 2790 exames, procedentes das Unidades Básicas de Saúde, Coordenadoria Regional de

\footnotetext{
${ }^{1}$ Acadêmico do $6^{\circ}$ ano de Medicina da Faculdade de Ciências Médicas da Santa Casa de São Paulo (FCMSCSP). E-mail: ricpira94@gmail.com

${ }^{2}$ Acadêmica do $6^{\circ}$ ano de Medicina da Faculdade de Ciências Médicas da Santa Casa de São Paulo (FCMSCSP). E-mail: amanda_frias@outlook.com

${ }^{3}$ Doutor em Medicina Preventiva, Faculdade de Medicina da USP. Professor Adjunto do Departamento de Saúde Coletiva da Faculdade de Ciências Médicas da Santa Casa de São Paulo (FCMSCSP). E-mail: nicarneirojr@uol.com.br.
}

Saúde Centro/SMS/SP. As variáveis selecionadas foram: tipo de exame, data de nascimento do usuário, tempo de espera da solicitação e hipótese diagnóstica. Utilizou-se Excel-Microsoft 2010 para armazenamento dos dados e confecção de relatórios. Dos exames, $73 \%$ apresentaram espera de 6 a 12 meses para realização, sendo a maioria dos beneficiados, mulheres entre 18 a 50 anos. As ultrassonografias foram majoritariamente as mais solicitadas, representando $88 \%$ do total. Dentre elas, a mamária bilateral foi predominante, com hipóteses diagnósticas não justificadas. Evidencia-se a utilização inadequada de exame nas consultas médicas da atenção primária, o que reforça a importância de protocolos clínicos, contribuindo na prevenção de iatrogenias e uma racionalidade eficiente do sistema de saúde. 
Descritores: Sistemas de Saúde. Atenção Primária à Saúde. Exames Médicos. Gestão em Saúde. Educação Médica.

\section{Abstract}

The lack of careful request for complementary examinations in medical appointments became incompatible with the availability of the Unified Health System (SUS), generating long-term programming, which requires intervention strategies. In this sense, in 2017, the Corujão da Saúde Program was created at the Municipal Health Secretariat of São Paulo (SMS / SP), in order to free the waiting lines for certain exams requested in the care network. Primary This cross-sectional and quantitative work characterized the relevance of these requests. A total of 2,790 examinations of the Basic Health Units of the Regional Health Coordination Center / SMS / SP were analyzed. The selected variables were: type of exam, date of birth of the user, waiting time for the request and diagnostic hypothesis. Excel-Microsoft 2010 was used for data storage and reporting. Of the exams, $73 \%$ had to wait between 6 and 12 months to complete, and most beneficiaries were women between 18 and 50 years. Ultrasounds were the most requested, representing $88 \%$ of the total. Among them, the bilateral breast predominated, with unjustified diagnostic hypotheses. The inadequate use of the test in primary care medical appointments is evident, which reinforces the importance of clinical protocols, contributing to the prevention of iatrogenesis and an efficient rationality of the health system.

Keywords: Health Systems. Primary Health Care. Medical Examination. Health Management. Medical Education.

\section{Resumen}

La falta de una solicitud cuidadosa de exámenes complementarios en las citas médicas se volvió incompatible con la disponibilidad del Sistema Único de Salud (SUS), generando una programación a largo plazo, que requiere estrategias de intervención. En este sentido, en 2017, se creó el Programa Corujão da Saúde en la Secretaría Municipal de Salud de São Paulo (SMS / SP), con el fin de liberar las líneas de espera para ciertos exámenes solicitados en la red de atención. Primaria Este trabajo transversal y cuantitativo caracterizó la relevancia de estas solicitudes. Se analizaron un total de 2.790 exámenes de las Unidades Básicas de Salud del Centro Regional de Coordinación de Salud / SMS / SP. Las variables seleccionadas fueron: tipo de examen, fecha de nacimiento del usuario, tiempo de espera para la solicitud e hipótesis de diagnóstico. Excel-Microsoft 2010 se utilizó para el almacenamiento de datos e informes. De los exámenes, el $73 \%$ tuvo que esperar entre 6 y 12 meses para completar, y la mayoría de los beneficiarios fueron mujeres entre 18 y 50 años. Los ultrasonidos fueron 
los más solicitados, representando el $88 \%$ del total. Entre ellos, predominó el seno bilateral, con hipótesis diagnósticas injustificadas. El uso inadecuado de la prueba en las citas médicas de atención primaria es evidente, lo que refuerza la importancia de los protocolos clínicos, contribuyendo a la prevención de la iatrogénesis y una racionalidad eficiente del sistema de salud.

Descriptores: Sistemas de Salud. Atención Primaria de Salud. Exámenes Médicos. Gestión en Salud. Educación Médica.

\section{Introdução}

A solicitação cada vez mais frequente de exames complementares de diagnóstico, especialmente os de imagens, tem se tornado incompatível com a disponibilidade de oferta, particularmente no setor público, no caso brasileiro o Sistema Único de Saúde (SUS). No âmbito do SUS o município de São Paulo atingiu a casa dos 485,3 mil pedidos, gerando "filas de espera" para realizações, sendo composta por: 349,2 mil ultrassonografias (USG), 42,2mil tomografias (TC), 33,5 mil ecocardiografias $\quad(\mathrm{ECO}), \quad 32,2 \quad \mathrm{mil}$ mamografias (MMG) e 28,1 mil ressonâncias $(\mathrm{RNM})^{1}$.

Com o intuito de responder a essa demanda reprimida, a gestão municipal do SUS de São Paulo, em janeiro de 2017, criou o Programa "Corujão da Saúde", com meta de zerar essa fila em até 90 dias. Para isso, organiza dois grupos de usuários: aqueles em que o pedido de exame havia sido feito em menos de 6 meses, representando 60\% (296,5 mil) dessa fila; e os que haviam excedido esse prazo $^{1}$.

O primeiro grupo - menos de 6 meses desde a solicitação do exame - foi contatado e agendado, boa parte em horários noturnos, e os exames foram realizados nos serviços municipais, como também em hospitais filantrópicos, por meio de convênios ${ }^{1}$.

Já para as pessoas que aguardavam há mais de 180 dias, serão agendadas uma nova consulta médica nas Unidades Básicas de Saúde (UBS) de origem². Nessa reconsulta, será avaliada a pertinência dos exames solicitados e a necessidade de outros encaminhamentos naquele momento: “o objetivo é verificar seus atuais quadros de saúde e a necessidade de serem submetidos ao exame originalmente prescrito ou a outros procedimentos".

Por meio do "Corujão da Saúde", espera-se aproximar ao padrão de solicitação do procedimento de no máximo 30 dias ${ }^{1}$. Esse prazo garante maior segurança ao paciente, pois logo após ter sido submetido a uma avaliação médica realizará um exame específico para a sua demanda, garantindo maior resolubilidade e satisfação.

Essa superfluidade pode advir da melhora do quadro clínico do paciente ou pela simples necessidade de realização de um 
procedimento distinto. Mais do que isso, esse limite de tempo também pode evitar iatrogenia, uma vez que restringe a realização de procedimentos, que por si só apresentam um risco intrínseco, que não são mais pertinentes depois de decorrido mais de um mês de consulta ${ }^{3}$.

Esse programa da Prefeitura de São Paulo poderá evidenciar também uma questão muito debatida ultimamente: a prescrição de exames complementares de maneira exagerada e sem um embasamento científico. Pesquisa realizada em Ribeirão Preto/São Paulo, evidenciou esse problema na rede pública de saúde, isto é, analisando uma amostra com 293 pacientes internados em um serviço hospitalar, entre 2006 e 2009, observaram que dos 9.522 exames laboratoriais realizados $(32,5$ exames para cada paciente), 568 (6\%) foram considerados desnecessários. Contatando-se, assim, solicitações não condizentes com os protocolos do SUS 3 .

A fim de enfrentar essa questão das solicitações de exame de maneira não criteriosa, tem-se recomendado os guidelines. Modelos estes criados e estabelecidos por equipes multidisciplinares, que baseados em revisões sistemáticas, têm a capacidade de indicar os melhores e mais adequados procedimentos para cada situação, considerando-se os riscos, benefícios e custos $^{3}$.
Os avanços na tecnologia médica, particularmente nos procedimentos diagnósticos, têm fortes impactos nos recursos orçamentários públicos, particularmente na nossa realidade, sendo um grande desafio a ser transposto, isto é, a insuficiência de financiamento para garantir a universalidade e a integralidade. Para ilustrar essa questão, pode-se fazer uma breve análise dos dados de 2011, no qual o gasto público brasileiro em saúde (União, estados e municípios) foi de 3,84\% do Produto Interno Bruto (PIB), enquanto a média dos países europeus com sistemas universais foi de $8,3 \%$ do PIB. Isso deixa clara a dificuldade do SUS em realizar suas ações e serviços a contento. Nesse sentido, o fenômeno dos exames complementares de diagnóstico desnecessários traz importante impacto nos sistemas de saúde ${ }^{4-5}$.

Muito além de questões financeiras, deve-se atentar ao fato de que o excesso de exames complementares não é isento de riscos ao paciente, podendo resultar no que se denomina de iatrogenia clínica, isto é, diz respeito aos potenciais danos biológicos e/ou psíquicos causados pela intervenção médica no indivíduo. Esse fenômeno tem tomado proporções significativas na assistência à saúde, sendo considerada a terceira maior causa de morte nos Estados Unidos da América do Norte ${ }^{6}$.

Alguns fatores que contribuem para esse aumento de exames podem estar 
associados a pressão dos próprios pacientes, motivados, entre outros aspectos, pela cultura de consumo de atos médicos impulsionados pelo mercado e a não integralidade do raciocínio clínico por parte do médico, orientado por uma formação hospitalocêntrica, com forte influência de uma prática médica apoiada em procedimentos especializados $^{6}$. Exames complementares auxiliam na composição de um diagnóstico de certeza, o que mostra que quando solicitados precisamente, são uma ferramenta excelente para ajudar o médico em sua hipótese diagnóstica.

As principais ferramentas de que o médico dispõe para fazer o diagnóstico do paciente são anamnese e exame clínico. Um estudo realizado na Inglaterra, em 1970, demonstrou que a anamnese isolada era responsável por $82,5 \%$ dos diagnósticos, anamnese mais exame físico por $91,25 \%$ e que anamnese, exame físico e exames complementares compunham $100 \%$ do diagnóstico. Um outro estudo realizado pelo de seis meses pela Atenção Básica de Saúde no contexto do Programa "Corujão da Saúde" da Secretaria Municipal de Saúde de São Paulo"*, caracterizando tipo de exame e associação com a hipótese diagnóstica que justifica o pedido, analisando, assim, sua pertinência no contexto da atenção básica em saúde, em município de grande porte, isto é, com mais de 100 mil habitantes ${ }^{8}$.
Hospital das Clínicas da Universidade de São Paulo, mostrou que uma história clínica e um exame físico bem feito são suficientes por realizar cerca de $70 \%$ dos diagnósticos, enquanto que os exames complementares, nos quais estão incluídos os exames de imagem, são responsáveis por apenas $30 \%$ dos diagnósticos. Assim sendo, o uso do raciocínio clínico composto por anamnese e exame físico auxilia no direcionamento de exames mais adequados para a hipótese diagnóstica, diminuindo, assim, a solicitação descriteriosa dos mesmos ${ }^{7}$.

Diante dessas questões apresentadas, consideramos oportuno analisar os exames complementares, objeto do Programa “Corujão da Saúde” da SMS-SP, particularmente os que estão com período de espera para realização igual ou maior de 180 dias. Esse artigo visa apresentar parte dos resultados da pesquisa "Análise da pertinência dos exames complementares solicitados há mais

\section{Método}

Estudo descritivo, transversal e de abordagem quantitativa ${ }^{9}$, realizado no período de setembro de 2017 a agosto de 2018, após divulgação dos dados do Programa, em 11/09/2017, e executado de acordo com as etapas do cronograma do projeto de pesquisa. Elege-se, por motivo de viabilidade operacional, a rede básica da Coordenadoria Regional de Saúde Centro (CRSCentro), 
Revista Eletrônica Gestão \& Saúde ISSN: 1982- 4785 Souza RMC; Frias A; Junior NC.

instância de gestão local da SMS/SP, com 09

Unidades Básicas de Saúde (UBS).

A lista de pacientes em espera para a realização dos exames complementares elegíveis pelo Programa "Corujão da Saúde" e as respectivas informações necessárias foram fornecidas pelo "setor de regulação" regional (CRSCentro), como também pelo nível central da SMS-SP. Um "banco de dados" em Excel-Microsoft 2010 foi organizado pelos pesquisadores, possibilitando a digitação padronizada e

\section{Resultado}

Estudo Dos Exames Complementares Solicitados... evitando a duplicidade de entrada a partir do número do Cartão Nacional de Saúde dos usuários

Para os objetivos desse artigo, as variáveis selecionadas de análise foram: tipos de exame; tempo de espera para realização do exame; hipótese diagnóstica e faixa etária dos pacientes. Outras variáveis, como dados sociais, identificação do estabelecimento que realizou os exames, entre outros, foram considerados não aplicáveis para o escopo deste artigo.

Tabela 1: Tipo e quantidade de procedimentos solicitados pelas UBS da CRS Centro, SMS-SP, no âmbito do Programa "Corujão da Saúde", período de 17/03/2015 a 19/07/2016

\begin{tabular}{|c|c|c|}
\hline Procedimentos & Quantidade & $\%$ \\
\hline ECOCARDIOGRAFIA TRANSTORACICA & 31 & $1,11 \%$ \\
\hline MAMOGRAFIA BILATERAL PARA RASTREAMENTO & 162 & $5,81 \%$ \\
\hline RESSONANCIA MAGNETICA DE BACIA / PELVE & 2 & $0,07 \%$ \\
\hline RESSONANCIA MAGNETICA DE COLUNA CERVICAL & 1 & $0,04 \%$ \\
\hline RESSONANCIA MAGNETICA DE COLUNA LOMBO-SACRA & 6 & $0,22 \%$ \\
\hline RESSONANCIA MAGNETICA DE CRANIO & 3 & $0,11 \%$ \\
\hline RESSONANCIA MAGNETICA DE MEMBRO SUPERIOR (UNILATERAL) & 2 & $0,07 \%$ \\
\hline RESSONANCIA MAGNETICA DE SELA TURCICA & 1 & $0,04 \%$ \\
\hline TOMOGRAFIA COMPUTADORIZADA DE ABDOMEN SUPERIOR & 4 & $0,14 \%$ \\
\hline TOMOGRAFIA COMPUTADORIZADA DE ARTICULACOES DE MEMBRO INFERIOR & 5 & $0,18 \%$ \\
\hline TOMOGRAFIA COMPUTADORIZADA DE ARTICULACOES DE MEMBRO SUPERIOR & 2 & $0,07 \%$ \\
\hline TOMOGRAFIA COMPUTADORIZADA DE COLUNA CERVICAL CIOUS/ CONTRASTE & 9 & $0,32 \%$ \\
\hline TOMOGRAFIA COMPUTADORIZADA DE COLUNA LOMBO-SACRA C/OUS/ CONTRASTE & 26 & $0,93 \%$ \\
\hline TOMOGRAFIA COMPUTADORIZADA DE COLUNA TORACICA CI OUS/ CONTRASIE & 2 & $0,07 \%$ \\
\hline TOMOGRAFIA COMPUTADORIZADA DE FACE / SEIOS DA FACE/ARTICULACOES TEMPORO-MANDIBULARES & 13 & $0,47 \%$ \\
\hline TOMOGRAFIA COMPUTADORIZADA DE PELVE/BACIA / ABDOMEN INFERIOR & 1 & $0,04 \%$ \\
\hline TOMOGRAFIA COMPUTADORIZADA DE PESCOCO & 2 & $0,07 \%$ \\
\hline TOMOGRAFIA COMPUTADORIZADA DE TORAX & 15 & $0,54 \%$ \\
\hline TOMOGRAFIA COMPUTADORIZADA DO CRANIO & 48 & $1,72 \%$ \\
\hline TOMOGPAFIA POR EMISSĀO DE POSITRONS (PET-CT) & 1 & $0,04 \%$ \\
\hline ULTRA-SONOGRAFIA DAS PARTES MOLES & 88 & $3,15 \%$ \\
\hline ULTRA-SONOGRAFIA DEABDOMEN SUPERIOR (FIGADO, VESICULA, VIAS BIUIARES) & 17 & $0,61 \%$ \\
\hline ULTRA-SONOGRAFIA DE ABDOMEN TOTAL & 186 & $6,67 \%$ \\
\hline ULTRA-SONOGRAFIA DE APARELHO URINARIO & 55 & $1,97 \%$ \\
\hline ULTRA-SONOGRAFIA DE ARTICULACAO & 45 & $1,61 \%$ \\
\hline ULTRA-SONOGRAFIA DE BOLSA ESCROTAL & 11 & $0,39 \%$ \\
\hline ULTRA-SONOGRAFIA DE PROSTATA & 91 & $3,26 \%$ \\
\hline UITRA-SONOGRAFIA DE TIREOIDE & 101 & $3,62 \%$ \\
\hline ULTRA-SONOGRAFIA DOPPLER COLORIDO DE VASOS ( ATE 3 VASOS ) & 34 & $1,22 \%$ \\
\hline ULTRA-SONOGRAFIA MAMARIA BILATERAL. & 1087 & $38,96 \%$ \\
\hline ULTRA-SONOGRAFIA OBSTETRICA & 169 & $6,06 \%$ \\
\hline ULTRA-SONOGRAFIA OBSTEIRICA MORFOLOGICA & 4 & $0,14 \%$ \\
\hline . & 13 & $0,47 \%$ \\
\hline ULTRA-SONOGRAFIA TRANSFONTANELA & 1 & $0,04 \%$ \\
\hline ULTRA-SONOGRAFIA TRANSVAGINAL & 552 & $19,78 \%$ \\
\hline Total & 2790 & $100,00 \%$ \\
\hline \multicolumn{3}{|l|}{ Fonte:CRS Centro, SMS-SP. } \\
\hline
\end{tabular}


Revista Eletrônica Gestão \& Saúde ISSN: 1982- 4785 Souza RMC; Frias A; Junior NC.

CRSCentro, elegíveis no Programa "Corujão da Saúde"/SMS/SP, no período de implantação desse Programa entre 10/01/2017 a 10/04/2017 com espera de 180 dias ou mais para a realização, correspondendo a 2759 indivíduos. Os exames incluídos foram: Ultrassonografia (USG), Ressonância Magnética Nuclear A USG mamária bilateral corresponde a cerca de $40 \%$ do total solicitado (Tabela 1 ).

Em relação ao ciclo de vida dos pacientes (Tabela 2), constatamos a presença importante de adultos (21-59 anos) $(41,86 \%)$, seguido por idosos (60 anos ou mais) $(12,72 \%)$. Crianças (1-10 anos) e adolescentes

Dos 3 procedimentos mais solicitados em cada faixa etária (Tabela 2), encontramos a seguinte distribuição: 1-10 anos o exame mais solicitado foi a USG de partes moles, seguido da USG mamária bilateral e da USG de abdome total. Já para a faixa etária de 11 a 40 anos os exames foram a USG mamária bilateral, a USG transvaginal e a USG obstétrica. Dos 41 aos 90 anos, houve um resultado idêntico dos dois
Estudo Dos Exames Complementares Solicitados... (RMN), Tomografia Computadorizada (TC), Ecocardiografia (ECO) e Mamografia (MMG). A Densitometria Óssea (DO). Todavia, a DO não foi observada na lista dos procedimentos solicitados pelas UBS.O tempo de espera para o acesso ao exame data de 17/03/2015 a 19/07/2016.

(11-20 anos) representaram 2,46\% desse universo. Deve-se, aqui, registrar o fato de que $1185(42,95 \%)$ pacientes não tinham sua data de nascimento disponível, logo, foram agrupados como "Sem Informações” (Si).

a USG mamária bilateral e a USG transvaginal. Porém, o terceiro exame mais frequente variou, isto é, dos 41 aos 50 anos temos USG de abdome total; dos 51 aos 60 anos USG de tireoide; dos 61 aos 70 anos USG de próstata; dos 71 aos 80 anos USG de tireoide e dos 81 aos 90 anos houve a mesma quantidade de solicitação para USG de partes moles, USG de aparelho urinário e TC de coluna lombo-sacra. primeiros exames

mais prescritos,

sendo

respectivamente:
Tabela 2: Distribuição quanto à faixa etária dos pacientes das UBS da CRS Centro, SMS-SP, que realizaram exames complementares incluídos no Programa "Corujão da Saúde", periodo de 17/03/2015 a 19/07/2016.

\begin{tabular}{|c|c|c|c|}
\hline & Faixa Etária & Número de pacientes & Porcentagem \\
\hline & 1-10 anos & 21 & $0,76 \%$ \\
\hline & 11-20 anos & 47 & $1,70 \%$ \\
\hline & 21-30 anos & 188 & $6,81 \%$ \\
\hline & $31-40$ anos & 309 & $11,20 \%$ \\
\hline & $41-50$ anos & 332 & $12,03 \%$ \\
\hline & $51-60$ anos & 326 & $11,82 \%$ \\
\hline & 61-70 anos & 240 & $8,70 \%$ \\
\hline & $71-80$ anos & 92 & $3,33 \%$ \\
\hline & 81- 90 anos & 16 & $0,58 \%$ \\
\hline & $>90$ anos & 3 & $0,11 \%$ \\
\hline \multirow{3}{*}{ Rev. Gestão \& Saúde (Bras } & $\mathrm{Si}$ & 1185 & $42,95 \%$ \\
\hline & Total & 2759 & $100,00 \%$ \\
\hline & \multicolumn{3}{|c|}{ Fonte: CRS Centro, SMS-SP. } \\
\hline
\end{tabular}

Fonte: CRS Centro, SMS-SP. 
Procedimento solicitado por tempo de aguardo para realização.

Dos 2790 procedimentos analisados, apenas 1163 (41\%) apresentavam as datas em que foram solicitados e efetivamente realizados após a inclusão no Programa "Corujão da Saúde". Assim sendo, notou-se que $73 \%$ dos exames complementares prescritos tiveram uma demora de 6 meses a 364 dias para serem realizados; 25\% dos pacientes aguardaram entre 1 ano e 1 ano e meio na fila de espera e apenas $2 \%$ esperaram por mais de 1 ano e meio para a realização

(Tabela 3 e Gráfico 1)

Tabela 3: Distribuição de procedimentos solicitados de acordo com tempo para realização dos pacientes da UBS da CRS Centro, SMS-SP, no âmbito do Programa "Corujão da Saúde", no periodo de 17/03/2015 a 19/07/2016.

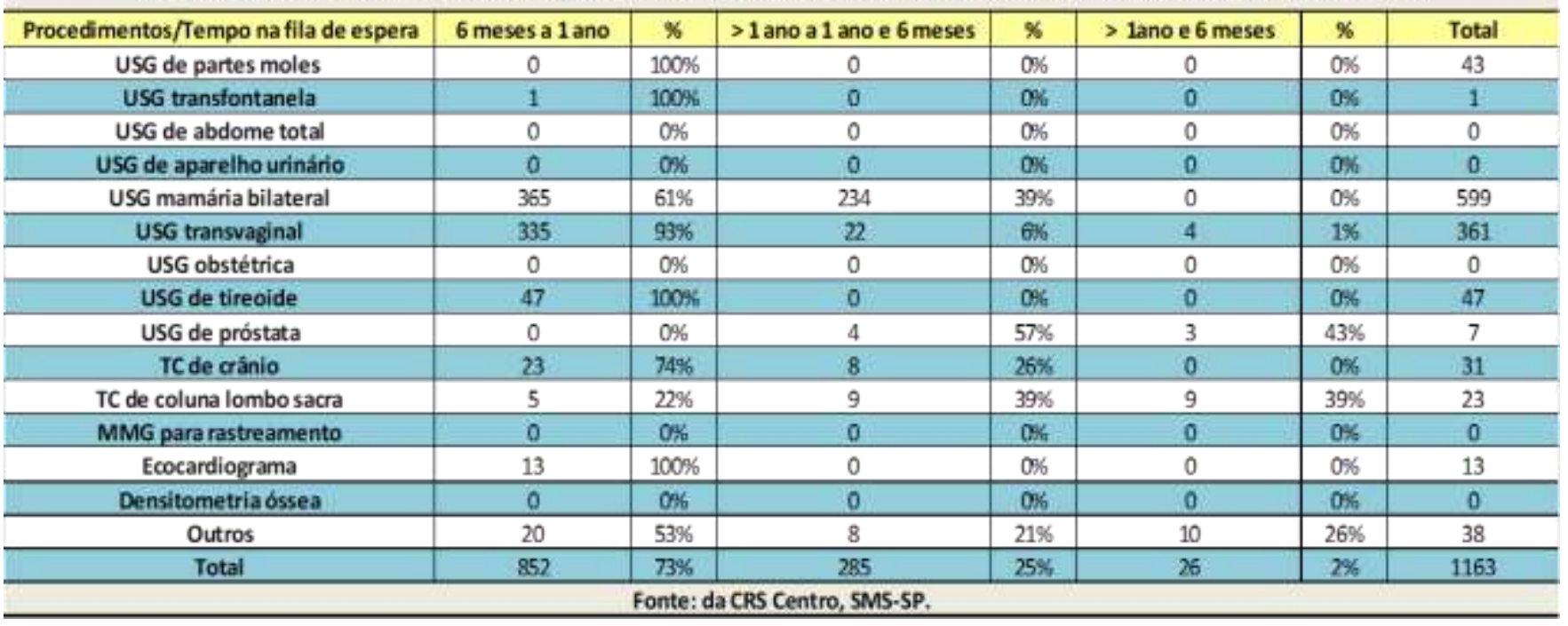

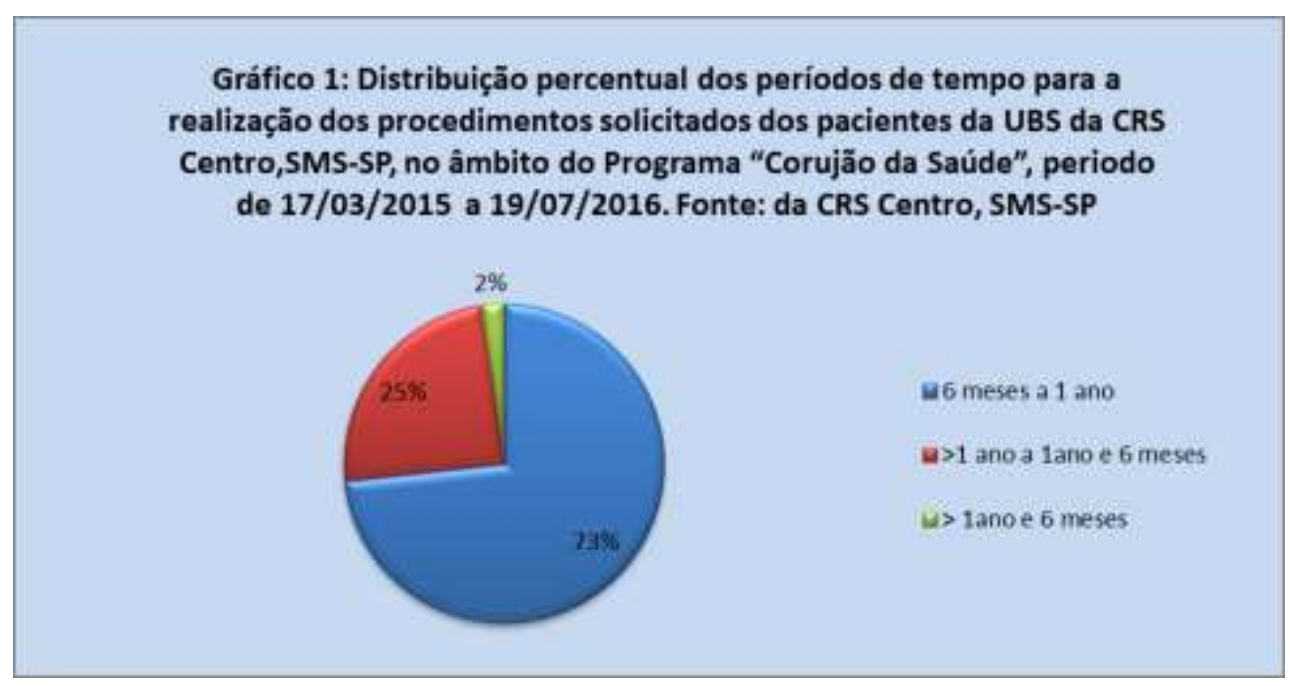

Quanto a associação entre hipóteses diagnósticas (HD) e procedimentos solicitados, $_{2}$ destacamos (Tabela 4) os principais procedimentos solicitados e as HD que foram registradas como justificativas.
Utilizou-se o CID 10 - Classificação Estatística Internacional de Doenças e Problemas Relacionados à Saúde, vol. 1, 10 a Revisão, 2012. 
Tabela 4: Distribuição do tipo de procedimento socilitado de acordo com as hipóteses diagnosticas dos pacientes das UBS da CRS Centro, SMSSP, no âmbito do Programa "Corujão da Saúde", no periodo de 17/03/2015 a 19/07/2016.

\begin{tabular}{|c|c|c|c|c|c|}
\hline Procedimento & CID-10 & Quantidade & $\%$ & Especificação & Total \\
\hline \multirow{8}{*}{ USG de partes moles } & $\mathrm{K} 40$ & 8 & $9 \%$ & Hérnia Inguinal & \multirow{8}{*}{88} \\
\hline & K42 & 4 & $5 \%$ & Hérnia Umbilical & \\
\hline & L72.0 & 4 & $5 \%$ & Cisto epidérmico & \\
\hline & D17.2 & 2 & $2 \%$ & Neoplasia lipomatosa benigna da pele e do tecido subcutâneo dos membros & \\
\hline & M25.5 & 2 & $2 \%$ & Dor articular & \\
\hline & M79 & 2 & $2 \%$ & Outros transtornos dos tecidos moles, não classificados em outra parte & \\
\hline & 200.0 & 2 & $2 \%$ & Exame geral e investigação de pessoas sem queixas ou diagnóstico relatado & \\
\hline & Outros/Si & 64 & $73 \%$ & Outros & \\
\hline USG transfontanela & A50.9 & 1 & $100 \%$ & Sifilis Congênita não especificada & 1 \\
\hline \multirow{4}{*}{ USG de abdome total } & R10 & 33 & $18 \%$ & Dor abdominal e pélvica & \multirow{4}{*}{186} \\
\hline & R10.4 & 8 & $4 \%$ & Outras dores abdominais e as não especificadas & \\
\hline & 200.0 & 2 & $1 \%$ & Exame geral e investigação de pessoas sem queixas ou diagnóstico relatado & \\
\hline & Outros/Si & 143 & $77 \%$ & Outros & \\
\hline \multirow{5}{*}{ USG de aparelho urinário } & N39 & 4 & $7 \%$ & Outros transtornos do trato urinário & \multirow{5}{*}{55} \\
\hline & N40 & 3 & $5 \%$ & Hiperplasia da próstata & \\
\hline & N20.9 & 3 & $5 \%$ & Calculose urinária, não especificada & \\
\hline & 200.0 & 2 & $4 \%$ & Exame geral e investigação de pessoas sem queixas ou diagnóstico relatado & \\
\hline & Outros/Si & 43 & $78 \%$ & Outros & \\
\hline \multirow{4}{*}{ USG mamária bilateral } & N63 & 287 & $26 \%$ & Nódulo mamário não especificado & \multirow{4}{*}{1087} \\
\hline & 201.4 & 104 & $10 \%$ & Exame ginecológico geral (geral) (de rotina) & \\
\hline & N95.1 & 91 & $8 \%$ & Estado de menopausa e de climatério feminino & \\
\hline & Outros/Si & 605 & $56 \%$ & Outros & \\
\hline \multirow{4}{*}{ USG transvaginal } & 201.4 & 152 & $28 \%$ & Exame ginecológico geral (geral) (de rotina) & \multirow{4}{*}{552} \\
\hline & N95.1 & 91 & $16 \%$ & Estado de menopausa e de climatério feminino & \\
\hline & R10.2 & 73 & $13 \%$ & Dor pélvica ou perineal & \\
\hline & Outros/Si & 236 & $43 \%$ & Outros & \\
\hline \multirow{4}{*}{ USG obstétrica } & 232.1 & 80 & $47 \%$ & Gravidez confirmada & \multirow{4}{*}{169} \\
\hline & 201.4 & 2 & $1 \%$ & Exame ginecológico geral (geral) (de rotina) & \\
\hline & 200.0 & 2 & $1 \%$ & Exame geral e investigação de pessoas sem queixas ou diagnóstico relatado & \\
\hline & $\mathrm{Si}$ & 85 & $50 \%$ & Outros & \\
\hline \multirow{5}{*}{ USG de tireoide } & E04.1 & 27 & $27 \%$ & Bócio não tóxico uninodular & \multirow{5}{*}{101} \\
\hline & E07.9 & 6 & $6 \%$ & Transtorno não especificado da tireoide & \\
\hline & E04.2 & 3 & $3 \%$ & Bócio não tóxico multinodular & \\
\hline & E03.9 & 3 & $3 \%$ & Hipotireoidismo não especificado & \\
\hline & Outros/Si & 62 & $61 \%$ & Outros & \\
\hline \multirow{4}{*}{ USG de próstata } & $\mathrm{N} 40$ & 19 & $21 \%$ & Hiperplasia da próstata & \multirow{4}{*}{91} \\
\hline & $\mathrm{Z12.5}$ & 18 & $20 \%$ & Exame especial de rastreamento de câncer de próstata & \\
\hline & N41 & 3 & $3 \%$ & Doenças inflamatórias da próstata & \\
\hline & Outros/Si & 51 & $56 \%$ & Outros & \\
\hline & $\mathrm{H} 9 \mathrm{O}$ & 6 & $13 \%$ & Perda de audição por transtorno de condução e/ou neurossensorial & \\
\hline & $H 66.3$ & 4 & $8 \%$ & Outras otites médias supurativas crônicas & \\
\hline TC de crânio & G43 & 4 & $8 \%$ & Enxaqueca & 48 \\
\hline & R51 & 3 & $6 \%$ & Cefaleia & \\
\hline & Outros/Si & 31 & $65 \%$ & Outros & \\
\hline & M51.1 & 9 & $35 \%$ & Trasntornos de discos lombares e de outros discos vertebrais com radicul opatia & \\
\hline & M54 & 3 & $12 \%$ & Dorsalgia & \\
\hline TC colunalombo-sacra & G55 & 3 & $12 \%$ & Compressões de rázes e dos plexos nervosos em doenças classificadas em outra parte & 26 \\
\hline 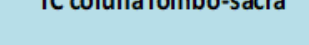 & M51 & 2 & $8 \%$ & Outros transtornos de discos vertebrais & \\
\hline & G55.1 & 2 & $8 \%$ & Compressões de rázes e dos plexos nervosos em transtornos dos discos vertebrais & \\
\hline & Outros/Si & 7 & $27 \%$ & Outros & \\
\hline & N63 & 14 & $9 \%$ & Nódulo mamário não especificado & \\
\hline MMG pararastreamento & N64.9 & 11 & $7 \%$ & Transtorno não especificado de mama & 160 \\
\hline Vivivo pard rastrearnento & N64 & 5 & $3 \%$ & Outras doenças de mama & 102 \\
\hline & Outros/Si & 132 & $81 \%$ & Outros & \\
\hline & 110 & 10 & $32 \%$ & Hipertensão Essencial (primária) & \\
\hline Ecocardiografia & 150.0 & 2 & $6 \%$ & Insuficiência cardíaca congestiva & 31 \\
\hline 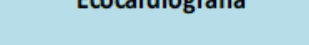 & 150 & 1 & $3 \%$ & Insuficiência cardiaca & \\
\hline & Outros/Si & 18 & $58 \%$ & Outros & \\
\hline Outros & Outros/Si & 193 & $100 \%$ & Outros & 193 \\
\hline & & & & & 2790 \\
\hline
\end{tabular}


A USG de partes moles está relacionada com a HD de "Hérnia Inguinal". A USG transfontanela teve associação com a HD de "Sífilis Congênita Não Especificada". Já para fundamentar a USG de abdome total temos HD "Dores Abdominais e Pélvicas". No caso da USG de aparelho urinário "Outros Transtornos de Aparelho Urinário" foi a primeira justificativa. Na USG mamária houve um predomínio de "Nódulo Mamário Não Especificado", enquanto na USG transvaginal "Exame Ginecológico Geral" (geral) (de rotina) foi a hipótese diagnóstica que mais foi apontada. Na USG obstétrica têm-se a HD "Gravidez Confirmada". Na USG de tireóide o "Bócio Não Tóxico Uninodular" foi a principal hipótese diagnóstica para solicitação de tal procedimento. No caso da USG de próstata a "Hiperplasia de Próstata" é a HD. "Perda de Audição por Transtornos de Condução e/ou Neurossensorial" foi a principal motivação para a prescrição TC de crânio. Para a TC de coluna lombo-sacra a hipótese mais utilizada foi "Transtornos de Discos Lombares e de Outros Discos Vertebrais com Radiculopatias". Já para a MMG de rastreamento, "Nódulos Mamários Não Especificados" foi apontada como a principal HD que justificava tal exame. As Ecocardiografias foram solicitadas fundamentalmente pela hipótese diagnóstica de "Hipertensão Essencial (primária)".

\section{Discussão}

A solicitação de exames complementares tem sido tema de amplo debate na atualidade da assistência médica, tanto no sistema público, como no privado $^{8}$. Essa preocupação tem se pautado, particularmente, na avaliação da pertinência, visando evitar prescrição indiscriminada, possíveis iatrogenias reflexas e gastos financeiros.

No contexto do sistema de saúde brasileiro, o número de procedimentos diagnósticos por consulta médica no SUS tem aumentado significativamente nas últimas décadas. Na região Sudeste, por exemplo, em 1995 era de 0,11 exames de imagem por consulta médica passando para 0,12 em 2000 e chegando a 0,14 em $2005^{9}$.

Tal fato nos leva a uma reflexão necessária de que a requisição de exames pelos médicos brasileiros vem se tornando cada vez mais exponencial, possivelmente pela formação hospitalocêntrica, centrada nas especializações e subespecializações, não considerando uma visão global e clínica baseada em evidências na assistência ao paciente ${ }^{6}$. Abordagem esta que é atributo da Atenção Primária à Saúde ${ }^{10}$.

Essas são questões presentes neste trabalho, quando analisamos no total de 2790 procedimentos que foram requisitados pelos médicos que atuam nas Unidades Básicas de Saúde localizadas na região centro da cidade de São Paulo.

Dentre essa vasta gama de procedimentos analisados, percebe-se um 
número muito expressivo de ultrassonografias, tendo a predominância da USG mamária bilateral, que representa mais de 14 vezes o número de mamografias, o segundo exame mais solicitado. Essa mesma relação, quando comparada aos dados gerais do município $^{11}$ é de aproximadamente 5,6 vezes, sendo solicitados, em 2017, cerca de 2,5 milhões de USG e um número ao redor de 440.000 MMG.

Em relação a faixa etária dos pacientes, destaca-se maior proporção de adultos (21 aos 59 anos), semelhantes com dados analisados da SMS/SP ${ }^{11}$. Esse resultado pode estar associado ao fato de que por volta dos 40 anos se iniciam os protocolos de rastreio para as doenças e comorbidades mais prevalentes. Segundo o Caderno de Atenção Primária - Rastreamento de $2010^{12}$, a única enfermidade que é prevalente nos adultos do sexo feminino e necessita de exame de imagem para sua detecção precoce é o Câncer de Mama. Esse dado coincide com o consenso das principais Sociedades Médicas Internacionais, que apontam a necessidade de rastreamento para os cânceres de Mama, Colo uterino e Colorretal. Para outros agravos, como o Câncer de Próstata, ainda não há evidência suficiente para que se institua um protocolo de rastreamento, seja com antígeno prostático específico (PSA) ou USG de próstata $^{12}$.

Por outro lado, é nesta faixa etária também em que aumenta a quantidade de pacientes hígidos que procuram por um atendimento médico para a realização de "check-up". Os problemas dessa rotina de exame são os resultados falsos positivos, que implicam muitas vezes em uma investigação mais apurada com exames mais dispendiosos e invasivos e até mesmo em tratamentos desnecessários e repercussões psicológicas. Há também os resultados falsos negativos, que erroneamente afastam a presença da doença rastreada, que se não acompanhado por um olhar clínico cuidadoso, pode incorrer em uma falsa sensação de segurança ao paciente, que pode vir a se descuidar com a sua saúde até o próximo "check-up"13.

Constata-se que as mulheres são as maiores usuárias dessas consultas médicas, corroborando com literatura específica que afirma na Atenção Básica 72,2\% dos pacientes são do sexo feminino ${ }^{14}$. A não presença dos homens nesses serviços é apontada por questões relacionadas à desvalorização do autocuidado e que os pacientes do sexo masculino não se sentem familiarizados ao ambiente da UBS, seja porque eles a consideram um ambiente feminilizado, por ser preferencialmente utilizado mulheres e onde o maior contingente de profissionais é também do sexo feminino ${ }^{15}$.

A expressiva quantidade de USG mamárias solicitada, observada em todas as faixas etárias, sendo a faixa de idade de 31 a 60 anos, com maior número (533 exames), não se apoia nas recomendações do Ministério da Saúde, como também nas Sociedades Médicas ${ }^{16}$. 
Esses exames de imagem das mamas têm grande importância clínicopreventiva se aplicados criteriosamente de acordo com as indicações específicas para cada faixa etária. Tal importância se dá pelo fato de haver uma alta prevalência do câncer de mama na população brasileira. Considerando-se apenas a população feminina, esse tumor é a segunda neoplasia maligna mais frequente, perdendo somente para o câncer de pele não melanoma ${ }^{16}$. Além disso, é a quinta causa de morte por câncer na população geral e a principal causa de óbito por câncer em mulheres no Brasil ${ }^{17}$. Por conta disso, seu rastreamento e diagnóstico precoce são protocolados por diretrizes do Ministério da Saúde ${ }^{12}$.

O rastreio do câncer de mama consiste na realização de mamografias bienais em mulheres de 50 a 69 anos. Estudos comprovam que, nesta faixa etária, métodos organizados de rastreamento fornecem à mulher um balanço favorável entre riscos e benefícios da sua realização. Já em outras idades e periodicidades, não há comprovação de maiores benefícios sobre riscos de se realizar o exame ${ }^{12}$. A mamografia é, portanto, o principal método de diagnóstico precoce do câncer de mama e vale ressaltar que nas mulheres que têm indicação clínica e realizam este exame na periodicidade adequada, ele representa a redução de $15 \%$ a $25 \%$ da mortalidade $^{17}$. Outros benefícios desta prevenção secundária é o melhor prognóstico da neoplasia, se identificada precocemente, e um tratamento subsequente mais efetivo. Já entre seus riscos, destacam-se os resultados falso-positivos, falso-negativos, sobre diagnóstico, sobre tratamento e a exposição à radiação ionizante ${ }^{12}$.

Por outro lado, a ultrassonografia mamária, que é um exame isento de radiação ionizante, não é utilizada como procedimento de rastreamento. Entre suas indicações está a complementação diagnóstica de mamografias inconclusivas e a utilização em mulheres com mamas densas ou com fatores de risco importantes para o câncer de mama, além de outras indicações importantes como o monitoramento de procedimentos invasivos e a avaliação de nódulos mamários ${ }^{18}$. Portanto, não são suficientes para justificar o elevado número deste tipo USG nos dados analisados ${ }^{13}$.

Outro dado que pode causar estranheza é a quantidade de MMG para rastreamento de CA de mama na fila de espera, que é irrisória se comparada com a quantidade de USG mamária solicitadas. Este fato pode ser explicado devido a maior facilidade de se realizar uma mamografia quando comparada à ultrassonografia, que é um exame examinador-dependente, ou seja, necessita de um profissional especializado para sua realização. Isto demanda maior tempo para conseguir realizá-lo e, por conseguinte, maiores filas de espera. Outro fato que pode explicar tal diferença é que a mamografia, por ser um exame de rastreio, apresenta maior disponibilidade às mulheres 
que utilizam a rede básica de saúde. Segundo a Secretaria de Estado da Saúde de São Paulo, as mulheres que se encontram dentro da faixa etária preconizada para rastreamento podem marcar suas mamografias sem necessidade de pedido médico.

Esta discrepância de solicitações entre MMG e USG não é exclusividade das UBS analisadas neste estudo. De acordo com dados do SIA-SUS (Sistema de Informação Ambulatorial), o número de ultrassonografias mamárias realizadas em mulheres na faixa etária entre 35 e 69 anos, no Brasil, nos anos 2014 a 2016 foi aproximadamente três vezes maior do que o número de mamografias na mesma faixa etária e período ${ }^{11}$.

O grande número de mulheres não incluídas na faixa etária preconizada para rastreamento para $\mathrm{CA}$ de mama e que realizaram USG mamária, também foi um dado de destaque. Pelo menos 450 exames deste tipo foram realizados em crianças, adolescentes e mulheres adultas menores de 50 anos, isto mostra que a grande maioria dos profissionais ainda não seguem protocolos do Ministério da Saúde e/ou de Sociedades de Especialista $^{16}$.

Além dos exames discutidos anteriormente, chama atenção a quantidade de USG transvaginal solicitada, isto é, mais de 500 pedidos. Porém, aproximadamente $30 \%$ das USG transvaginais foram justificadas por motivos de "rotina". O Ministério da Saúde, em sua publicação "Exames de Apoio Diagnóstico”, afirma que “...não foram encontradas indicações da realização do exame transvaginal como 'rotina' nas referências bibliográficas baseadas em

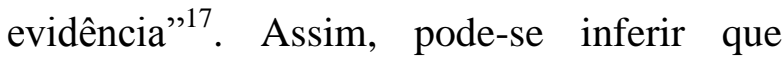
grandes partes dessas solicitações não se enquadram nas recomendações oficiais.

\section{Conclusão}

Constata-se que a questão da solicitação excessiva de exames complementares é algo que deve continuar a ser estudado e estar sempre entre as discussões dos órgãos gestores de saúde. Dado ao elevado número de procedimentos pedidos a cada consulta médica, as filas de espera para a realização de exames só aumentam com o passar do tempo, sendo inclusive citado que "o gargalo do SUS no município de São Paulo é a média complexidade" $"$. Em outras palavras, indica uma importante dificuldade para que o SUS possa se tornar mais resolutivo e garantir a integralidade. Será que o estabelecimento de protocolos supervisionados para a prescrição desses exames não ajudaria a frear essa solicitação indiscriminada? $\mathrm{O}$ fato de muitos destes procedimentos serem solicitados em função de um rastreamento ou "exame de rotina" não preconizado pelo Ministério da Saúde implica em uma não observância pelos médicos das boas práticas clínicas, baseadas em evidências. Tais práticas evitariam a maior oneração dos cofres públicos e chances de causar iatrogenias reflexas aos pacientes. Portanto, este trabalho traz não só potenciais 
benefícios para a organização da assistência à saúde na rede pública municipal de São Paulo, mas também, contribui para reflexões da própria formação médica, pois apontam questões a respeito de sua deficiência.

\section{Agradecimentos}

Aos funcionários e gerentes das Unidades Básicas de Saúde da Coordenadoria Regional de Saúde Centro (CRSCentro), da Secretaria Municipal de Saúde de São Paulo (SMS/SP); à Dra. Maria Emília Carvalhaes Machado (Setor de Regulação da CRSCentro); ao Dr. Sandro Garcia Hilário (Coordenador da Regulação da SMS/SP); ao Sr. Marcelo Alves da Silva (Assessoria Técnica de Tecnologia de Informação da SMS/SP); à Dra. Sonia Aparecida Alves (Coordenadora do Grupo Técnico de Regulação da Secretaria de Estado da Saúde de São Paulo) e ao Sr. Rodolfo M. C. de Souza. 


\section{Referências}

1. REFEITURA DA CIDADE DE SÃO PAULO. Prefeitura firma convênio para consultas de reavaliação do "Corujão da Saúde". São Paulo; 2017 Jan. Acesso 14/04/2017 em: capital.sp.gov.br/noticia/programa201ccorujao-da-saude201d-comeca-afuncionar-na-cidade.

2. Arena Thiago Roberto Castellane, Jericó Marli de Carvalho, Castro Liliana Cristina de, Castilho Valéria, Lima Antonio Fernandes Costa. Gastos com exames complementares desnecessários para hipertensos e diabéticos nos serviços de saúde. Rev. Gaúcha Enferm. [Internet]. 2014 Dec [acesso em 23 Jul 2018] ; 35( 4 ): 8693. Available fro Disponível em: http://www.scielo.br/scielo.php?script= sci_arttext\&pid=S1983-

14472014000400086\&lng=en.

3. Oosterhuis WP, Bruns DE, Watine J, Sandberg S, Horvath AR. EvidenceBased Guidelines in Laboratory Medicine: Principals and Methods. Clinical Chemistry. [Internet]. 2004 April [acesso em 28 Jul 2018] ; 50 (5): 806-818. Disponível em: http://clinchem.aaccjnls.org/content/50/ 5/806.long

4. Mendes Âquilas. A longa batalha pelo financiamento do SUS. Saude soc. [Internet]. $2013 \mathrm{Dec}$ [acesso em 17 Jul 2018] ; 22( 4 ): 987-993. Disponível em: http://www.scielo.br/scielo.php?script= sci_arttext\&pid=S0104$12902013000400002 \& \operatorname{lng}=$ en

5. Del Nero CR. O que é Economia da Saúde. In: Piola Francisco S., Vianna Solon M. (orgs). Economia da Saúde: conceitos e contribuição para a gestão da saúde. Brasília: IPEA, 2002. Pp 521.

6. Norman Armando Henrique, Tesser Charles Dalcanale. Prevenção quaternária na atenção primária à saúde: uma necessidade do Sistema Único de Saúde. Cad. Saúde Pública [Internet]. 2009 Sep [acesso em 28 Jul 2018] ; 25 ( 9 ): 2012-2020. Disponível em: http://www.scielo.br/scielo.php?script= sci_arttext\&pid=S0102-

311X2009000900015\&lng=en

7. Benseñor I. Anamnese, exame clínico e exames complementares como testes diagnósticos. revistadc [Internet]. 2013 [acesso em 28 Jul 2018]; 92(4):236-41. Disponível em:http://www.revistas.usp.br/revistad c/article/view/85896.

8. Capilheira MF, Santos IS. Epidemiologia da solicitação de exame complementar em consultas médicas. Rev. Saúde Pública [Internet]. 2006 [acesso em 27 Jul 2018]; 40 (2): 289$297 . \quad$ Disponível em: http://www.scielo.br/scielo.php?script= sci_arttext\&pid=S0034-

89102006000200015\&lng=en.

9. Ministério da Saúde. Indicadores básicos para a saúde no Brasil: conceitos e aplicações / Rede Interagencial de Informação para a Saúde - 2. ed.. Brasília. Organização Pan-Americana da Saúde [Internet], 2008 [acesso em 26 Jul 2018]. Disponível em http://tabnet.datasus.gov.br/tabdata/livr oidb/2ed/indicadores.pdf (pp. 279)3.

10. Agência Nacional de Saúde. Atributos da Atenção Primária à Saúde [Internet], 2018 [acesso em 25 Jul 2018]. Disponível em http://ans.gov.br/images/stories/Particit acao_da_sociedade/2018_GT_LabDipr o/CRIT\%C3\%89RIOS_DE_AVALAI \%C3\%87\%C3\%83O_-

_ATRIBUTOS_DA_ATEN\%C3\%87\% C3\%83O_PRIM\%C3\%81RIA_\%C3\% 80_SA\%C3\%9ADE.pdf.

11. Secretaria Municipal da Saúde. Coordenação de Epidemiologia e Informação - CEInfo. Boletim CEInfo Saúde em Dados | Ano XVII, n ${ }^{\circ}$ 17, Jun 2018. São Paulo (SP). Secretaria Municipal da Saúde [Internet], 2018 [acesso em 26 Jul 2018]. Disponível em:

http://www.prefeitura.sp.gov.br/cidade/ secretarias/upload/saude/arquivos/publi 
cacoes/Boletim_CEInfo_Dados_2018.p df.

12. Ministério da Saúde. Secretaria de Atenção à Saúde. Departamento de Atenção Básica. Caderno de Atenção Primária- Rastreamento. Brasília. Ministério da Saúde [Internet], 2010 [acesso em 28 Jul 2018]. Disponível em:

http://bvsms.saude.gov.br/bvs/publicac oes/caderno_atencao_primaria_29_rast reamento.pdf.

13. Martins MA. Check-up do check-up. Rev. Assoc. Med. Bras. [Internet]. 2005 [acesso em 28 Jul 2018]; 51 (3): 121-121. Disponível em:http://www.scielo.br/scielo.php?scr ipt=sci_arttext\&pid=S010442302005000300001\&lng=en.

14. Pimentel Í, Coelho B, Lima J, Ribeiro F, Sampaio F, Pinheiro R, Rocha-Filho F. Caracterização da demanda em uma Unidade de Saúde da Família. Revista Brasileira de Medicina de Família e Comunidade [Internet]. 2011; [acesso em 25 Jul 2018]; 6(20): 175-181. Disponível em: https://www.rbmfc.org.br/rbmfc/article /view/95.
15. Figueiredo W. Assistência à saúde dos homens: um desafio para os serviços de atenção primária. Ciênc. saúde coletiva [Internet]. 2005 [acesso em $27 \mathrm{Jul}$ 2018]; 10 (1): 105-109. Disponível em: http://www.scielo.br/scielo.php?script= sci_arttext\&pid=S1413$81232005000100017 \& \operatorname{lng}=\mathrm{en}$.

16. Instituto Nacional de Câncer José Alencar Gomes da Silva [homepage na internet]. Câncer da mama [acesso em 26 Jul 2018]. Disponível em: http://www.inca.gov.br.

17. Silva FX, Katz L, Souza ASR, Amorim MMR. Mamografia em mulheres assintomáticas na faixa etária de 40 a 49 anos. Rev. Saúde Pública [Internet]. 2014 [acesso em 27 Jul 2018]; 48 (6): 931-939. Disponível em: http://www.scielo.br/scielo.php?script= sci_arttext\&pid=S003489102014000600931\&lng=en

18. Vasconcelos, Rogerio Gonçalves; Uemura, Gilberto; Schirmbeck, Tarciso; Vieira, Karinie Marinho; Ultrassonografia mamária: aspectos contemporâneos / Breast sonography: contemporary aspects. Comun. ciênc. saúde;22(sup. espc. 1):129-140, 2011

\section{Participação dos autores:}

Souza RMC trabalhou na concepção teórica, coleta de dados, análise estatística e elaboração e redação final do texto.

Frias A trabalhou na concepção teórica, coleta de dados, análise estatística e elaboração e redação final do texto.

Junior NC trabalhou na concepção teórica, coleta de dados, análise estatística e elaboração e redação final do texto.
Recebido: 12.06.2019

Revisado: 13.06.2019

Aprovado: 13.09.2019 